\title{
Introduction for special issue
}

\section{"Epithelial regeneration in inflammatory diseases"}

\author{
Ryuichi Okamoto ${ }^{1,2, *}$
}

${ }^{1)}$ Department of Advanced Therapeutics in GI Diseases, and ${ }^{2)}$ Department of Gastroenterology and Hepatology, Graduate School, Tokyo Medical and Dental University, Tokyo, Japan.

Epithelial cells constitute the tissue structure and also play indispensable roles in various organs, including skin, lung, and the digestive tract. Besides acquiring tissue-specific functions, their common and most important role is to serve as a physical barrier between inner- and outer- environment. Upon inflammation of its resident tissue, the continuous structure of the epithelial layer is often disrupted, which results in loss of static environment in inner side of the tissue. Such a change in the inner environment may act as a key event to drive further exacerbation and persistence of the local inflammation. Thus, proper regeneration to restore the continuity of the epithelial layer is a prerequisite to give an end to the initiated inflammation. In this special issue, we would like to overview what we now know about how tissue regeneration is executed in various tissues, under inflammatory environment. Further studies may reveal how the inflammatory environment, both at cellular and molecular level, modulates tissue regeneration and give the basis for the establishment of therapeutic approach directed to tissue regeneration in various inflammatory diseases.

${ }^{*}$ Correspondence should be addressed to:

Ryuichi Okamoto, Department of Advanced Therapeutics in GI Diseases, and Department of Gastroenterology and Hepatology, Graduate School, Tokyo Medical and Dental University, 1-5-45 Yushima, Bunkyo-ku, Tokyo 113-8519, Japan. Tel: +81-3-5803-5974, Fax: +81-3-5803-0268, E-mail: rokamoto.gast@tmd.ac.jp

Key words:

Epithelial cells, stem cells, bone marrow derived cells, pro-inflammatory cytokines 


\section{Introduction}

The epithelial tissue constitutes the outer surface of the body, giving a physical margin between innerand outer-side of the body. Such an epithelial tissue is not only a component of the skin, a major tissue confronting the outer environment of the body, but also a component of organs inside the body, such as lung, uterus and the gastrointestinal tract.

Epithelial cells in each organ acquire distinct functions as they differentiate and mature, and subsequently play specific roles in their resident tissue. These tissue specific function of the epithelial tissue usually consists an indispensable part of the residing organ; i.e. nutrition absorption, gut-hormone secretion, or antimicrobial peptide secretion in the gastrointestinal tissue. However, a stable but ideal environment must be maintained within the inner side of the body, for the epithelial tissue to fully express their tissue specific functions. Thus the common and most important role of epithelial tissue over various organs may be its role as a physical barrier separating the unstable outer environment, and the stable inner environment.

Chronic inflammation may cause various diseases in each organ. Such an inflammation may be initiated by an unknown trigger, or by the damage of the epithelial tissue itself. Consequently, an ulcer may arise, where continuous integrity of the epithelial layer is disrupted, and where various external stimulants such as bacteria, viruses, various antigens or chemical compounds are allowed to easily access the underlying inner tissue. In general, such an influx of undesired stimulants contribute to the exacerbation and persistence of the local inflammation, by activating pro-inflammatory response of the underlying immune system. Thus, once inflammation is initiated, it cannot be ended without proper and complete regeneration of the epithelial tissue. In this special issue, we would like to provide overview of what we now know about how epithelial tissues in each organ executes such an important response in inflammatory diseases.

\section{Role of tissue-specific stem cells}

In the process of regeneration, existence of functional, tissue-specific stem cells may be a critical issue. As a rapid and abundant supply of newly generated epithelial cells is required to reconstruct the damaged tissue, the potential of stem cells to provide enough number of progenitor cells may limit the whole regenerative response ${ }^{1)}$. Thus, studies regarding the function of tissue stem cells under inflammatory environments may not only reveal the mechanism how stem cells contribute to the regeneration program, but also provide basis for regenerative therapies through manipulation of stem cell function. In this special issue, Fujishima et al reviews current studies regarding stem cells in the lung epithelium, and discuss its role in regeneration from acute and chronic inflammatory lung disease.

\section{Role of bone-marrow derived cells}

Studies have suggested that bone-marrow cells may have "plasticity" and thereby contribute to tissue repair in various organs, especially when tissue inflammation is present ${ }^{2), 3), 4)}$. Despite number of reports have suggested that such trans-differentiation may exist in a certain setting, its contribution to tissue regeneration still remains controversial ${ }^{5),}$ ). However, based on disease model studies, several clinical trials are on the way to evaluate the benefit of transplanting bone-marrow derived cells to treat non-hematopoietic diseases ${ }^{7), 8)}$. In this special issue, both Fujishima et al and Fujita et al overview the contribution of bone-marrow derived cells in lung and skin regeneration, respectively. Also, Fujita et al further refers to the clinical trial that is going on, and give prospects for its use in skin disorders.

\section{Crosstalk between molecular signaling pathways mediating inflammatory and regenerative response in epithelial cells}

As a result of tissue damage, the epithelial tissue must complete the regeneration program under the influence of inflammatory environment. Such environments include abundant stimuli from invaded pathogens, pro-inflammatory cytokines and undesired conditions such as hypoxia ${ }^{9), 10)}$. Thus studies aimed to reveal how epithelial cells, especially stem cells, cope with these unfavorable stimuli or conditions are interesting and also important ${ }^{11)}$. This issue will be discussed in the review regarding regeneration in inflammatory bowel diseases.

\section{Concluding remarks}

Through this special issue, we aimed to provide overview of present understandings upon tissue regeneration in inflammatory diseases. Future studies may further reveal cellular as well as molecular basis 
of tissue regeneration in various inflammatory diseases, and lead to establishment of novel therapeutic procedures that can be applied to treat severe or refractory tissue damages.

\section{References}

1) Bergmann A and Steller H: Apoptosis, stem cells, and tissue regeneration. Sci Signal. 2010; 3: 8.

2) $\mathrm{Wu} Y$, Zhao RC, Tredge EE: Concise review: bone marrow-derived stem/progenitor cells in cutaneous repair and regeneration. Stem Cells. 2010; 8: 905-915.

3) Kuçi S, Kuçi Z, Latifi-Pupovci H, Niethammer D, Handgretinger R, Schumm M, Bruchelt G, Bader P, Klingebiel T: Adult stem cells as an alternative source of multipotential (pluripotential) cells in regenerative medicine. Curr Stem Cell Res Ther. 2009; 4: 107-117.

4) Almeida-Porada G, Zanjani ED, Porada CD: Bone marrow stem cells and liver regeneration. Exp Hematol. 2010; 38: 574-580.

5) Lin F, Moran A, Igarashi P: Intrarenal cells, not bone marrow-derived cells, are the major source for regeneration in postischemic kidney. J Clin Invest. 2005; 115: 1756-1764.

6) Kavanagh DP, Kalia N: Hematopoietic Stem Cell Homing to Injured Tissues. Stem Cell Rev 2011; e-pub.

7) Saito $T$, Okumoto $K$, Haga $H$, Nishise $Y$, Ishii R, Sato C, Watanabe H, Okada A, Ikeda M, Togashi H, Ishikawa T, Terai S, Sakaida I, Kawata S: Potential Therapeutic Application of Intravenous Autologous Bone Marrow Infusion in Patients with Alcoholic Liver Cirrhosis. Stem Cells Dev. 2011; e-pub.

8) Malgieri A, Kantzari E, Patrizi MP, Gambardella S: Bone marrow and umbilical cord blood human mesenchymal stem cells: state of the art. Int J Clin Exp Med. 2010; 3: 248-269.

9) Melillo G: Hypoxia: jump-starting inflammation. Blood. 2011; 117: 2561-1562.

10) Eltzschig and Carmeliet: Hypoxia and inflammation. N Engl J Med. 2011; 364: 656-665.

11) Gómez-Nicola $D$, Valle-Argos B, Pallas-Bazarra $N$, Nieto-Sampedro M: IL-15 regulates proliferation and self-renewal of adult neural stem cells. Mol Biol Cell. 2011; e-pub. 